\title{
Supporting information A Real Colorimetric Thrombin Aptasensor By Masking Surfaces of Catalytically Active Gold Nanoparticles
}

Zhengbo Chen, * Lulu Tan, Liangyu Hu, Yimeng Zhang, Shaoxiong Wang, and Fanyi

$$
L v
$$

Department of Chemistry, Capital Normal University, Beijing, 100048, China

[*] Dr Zhengbo Chen, Zhanfang Ma, Corresponding author.

Tel+86-010-68903047.E-mail:czb979216@sina.com 


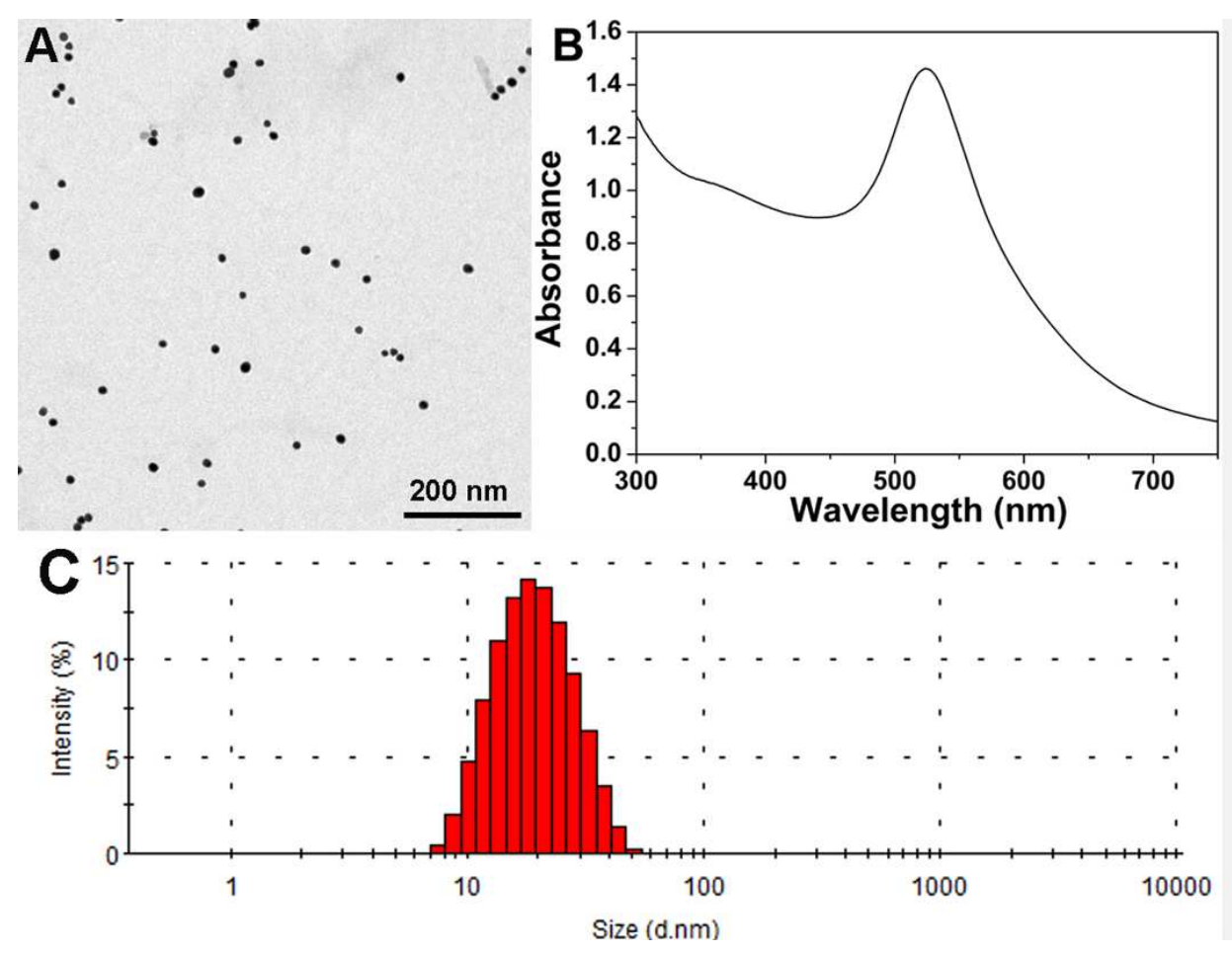

Fig. S1 TEM image (A), UV-Vis spectrum (B), and the particle size distribution (C) of AuNPs. 


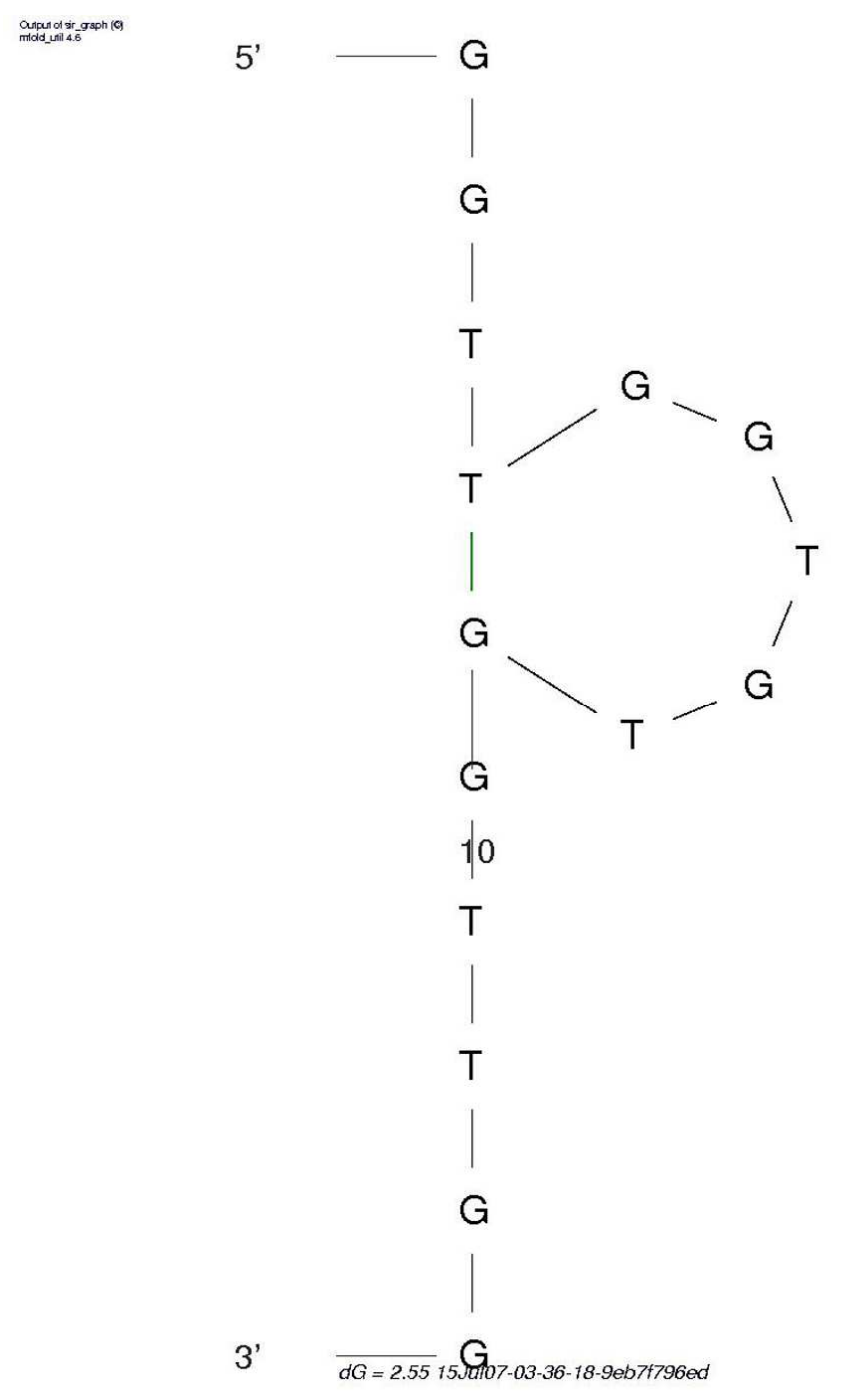

Fig. S2 Secondary structure of thrombin aptamer as predicted by M-Fold tool based on Zuker algorithm. 

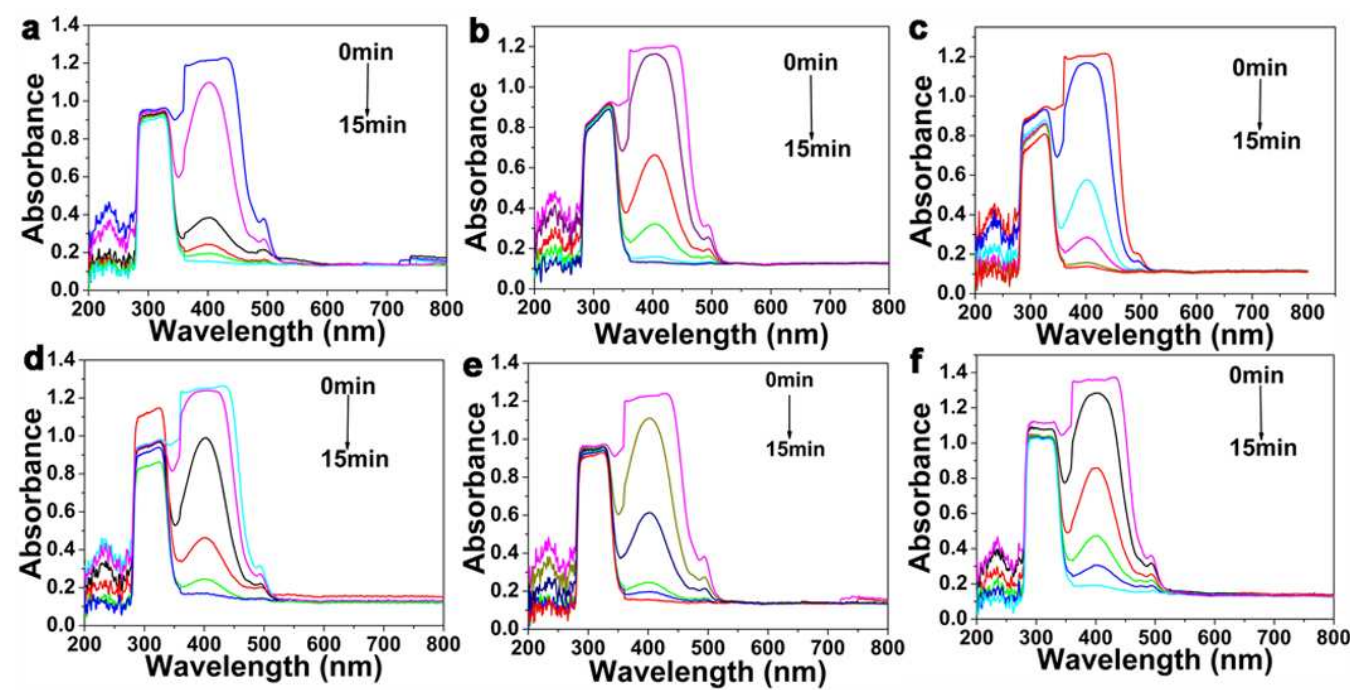

Fig. S3 Spectral changes showing the catalytic reduction of 4-nitrophenol to 4-aminophenol. TBA

concentration: (a) 0; (b) $10 \mathrm{nM}$; (c) $50 \mathrm{nM}$; (d) $100 \mathrm{nM}$; (e) $500 \mathrm{nM}$; and (f) $1 \mathrm{mM}$. 

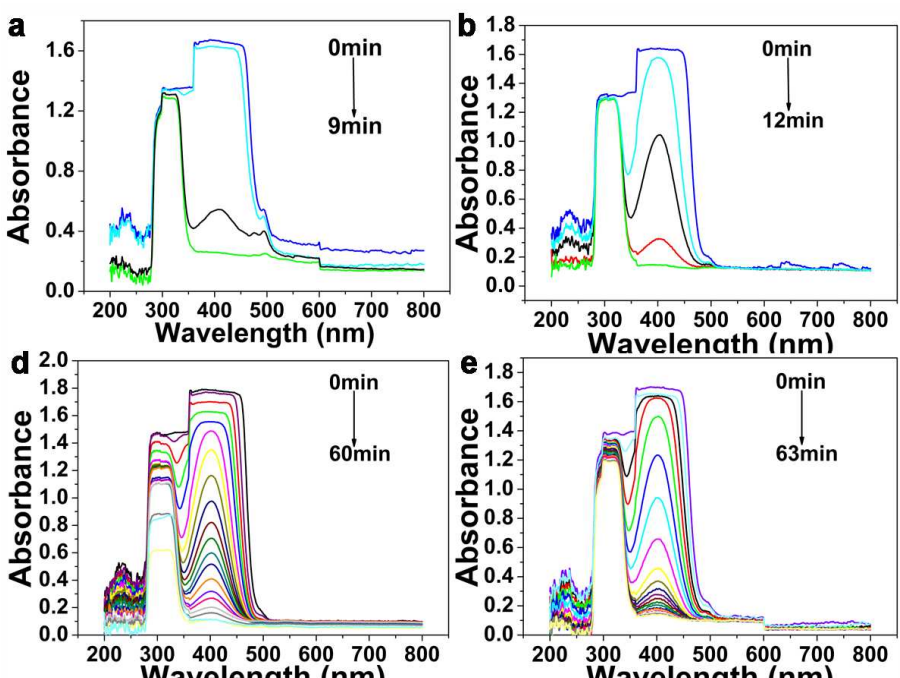

$300 \quad 400500600700$
Wavelength $(\mathrm{nm})$
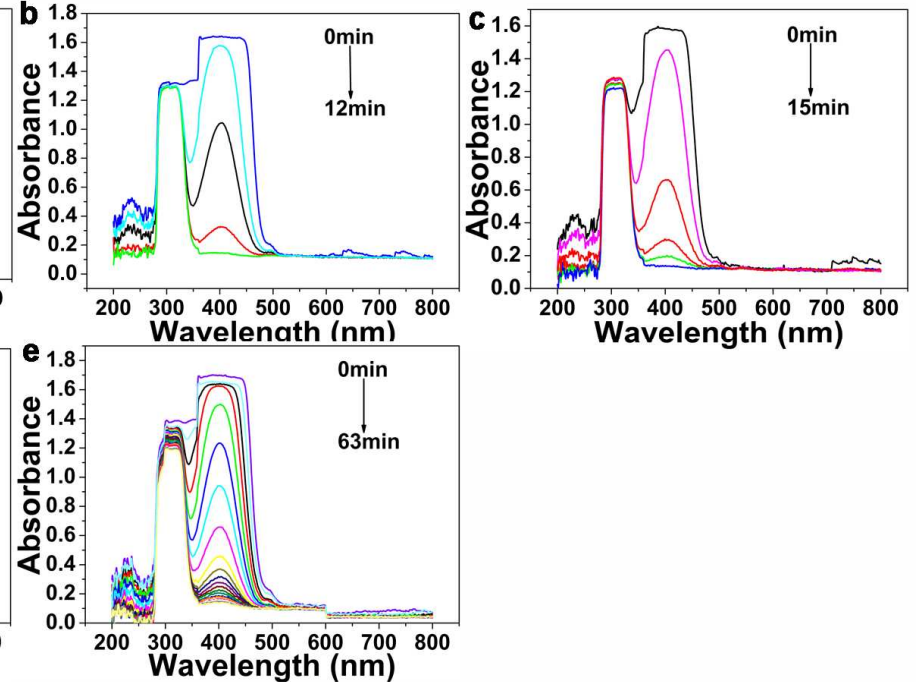

Fig. S4 Spectral changes showing the catalytic reduction of 4-nitrophenol to 4-aminophenol.

$\mathrm{NaH}_{2} \mathrm{PO}_{4}$ concentration: (a) 0; (b) $5 \mathrm{mM}$; (c) $10 \mathrm{mM}$; (d) $50 \mathrm{mM}$; and (e) $100 \mathrm{mM}$. 

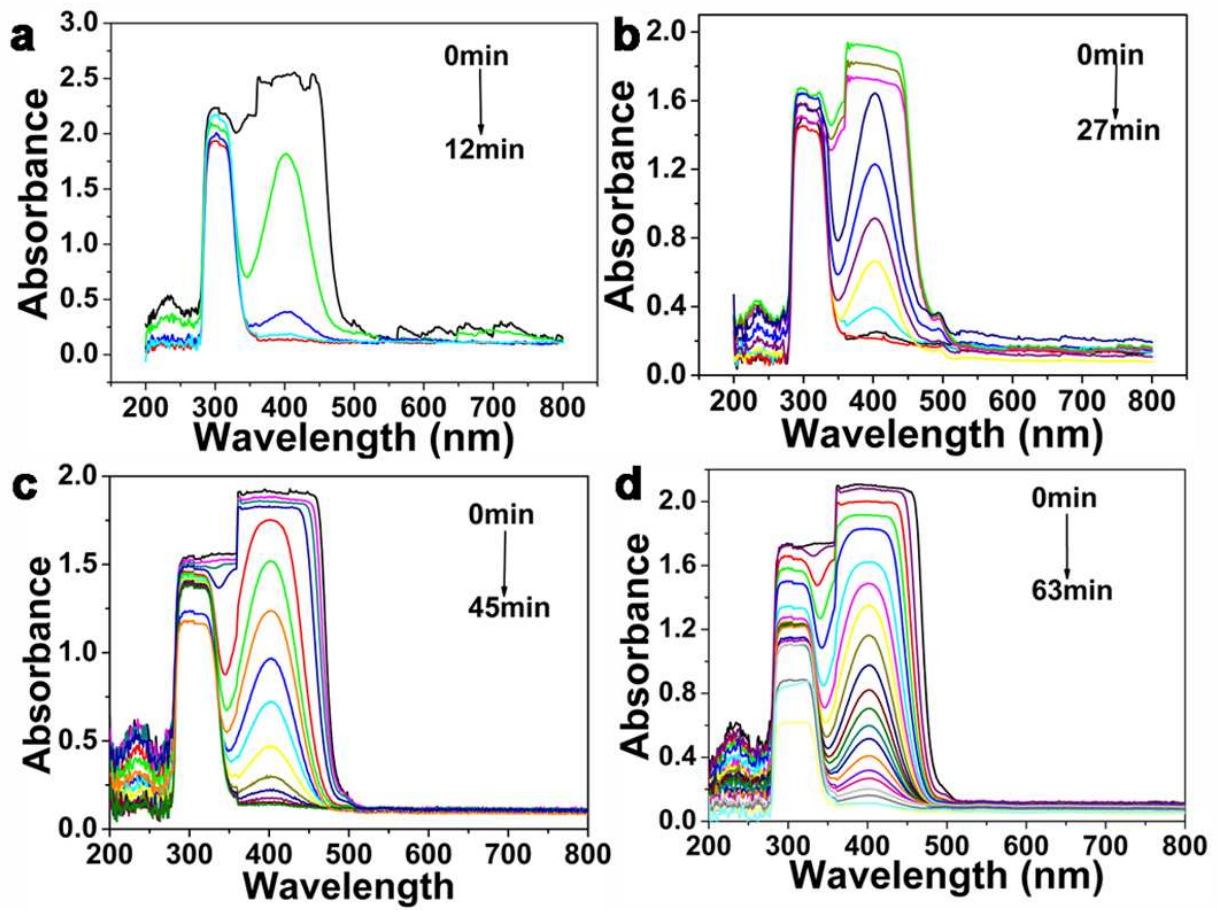

Fig. S5 Spectral changes showing the catalytic reduction of 4-nitrophenol to 4-aminophenol.

Aptamer incubation time: (a) 0; (b) $20 \mathrm{~min}$; (c) $40 \mathrm{~min}$; and (d) $60 \mathrm{~min}$. 

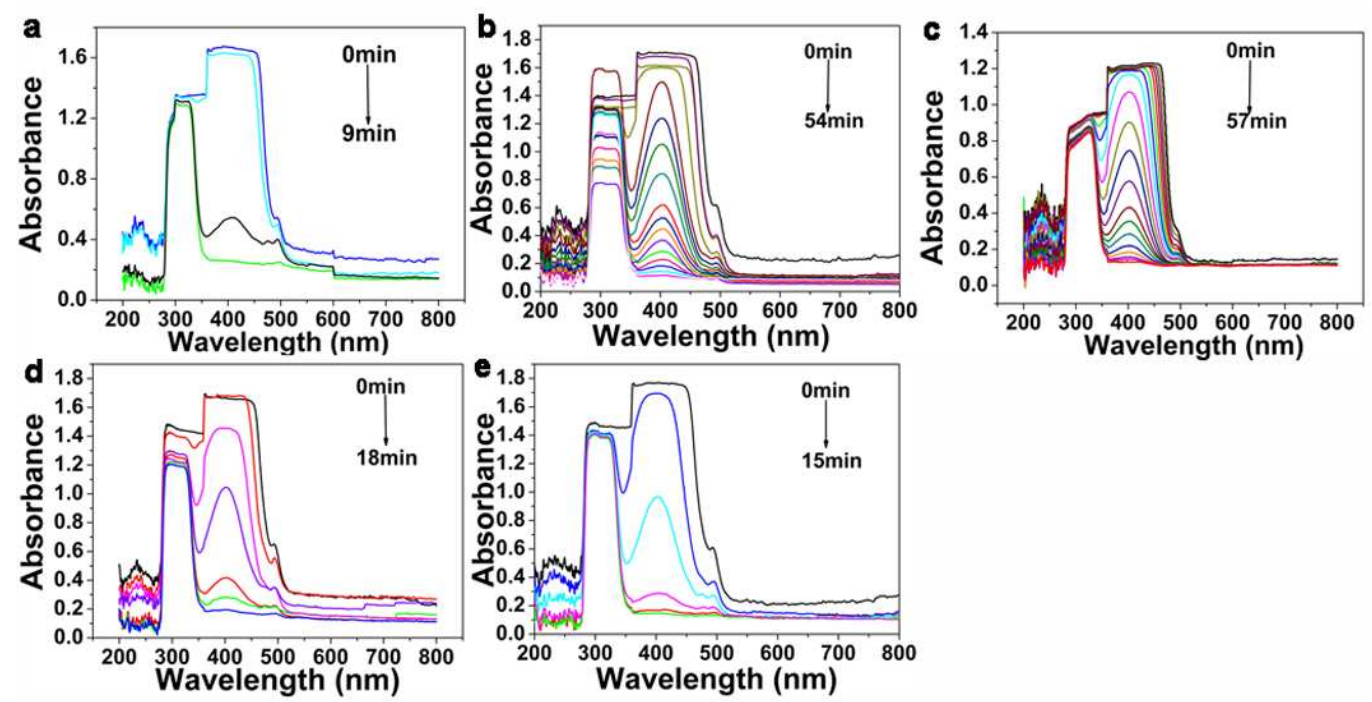

Fig. S6 Spectral changes showing the catalytic reduction of 4-nitrophenol to 4-aminophenol.

Thrombin incubation time: (a) 0; (b) $20 \mathrm{~min}$; (c) $40 \mathrm{~min}$; (d) $60 \mathrm{~min}$; and (e) $80 \mathrm{~min}$. 


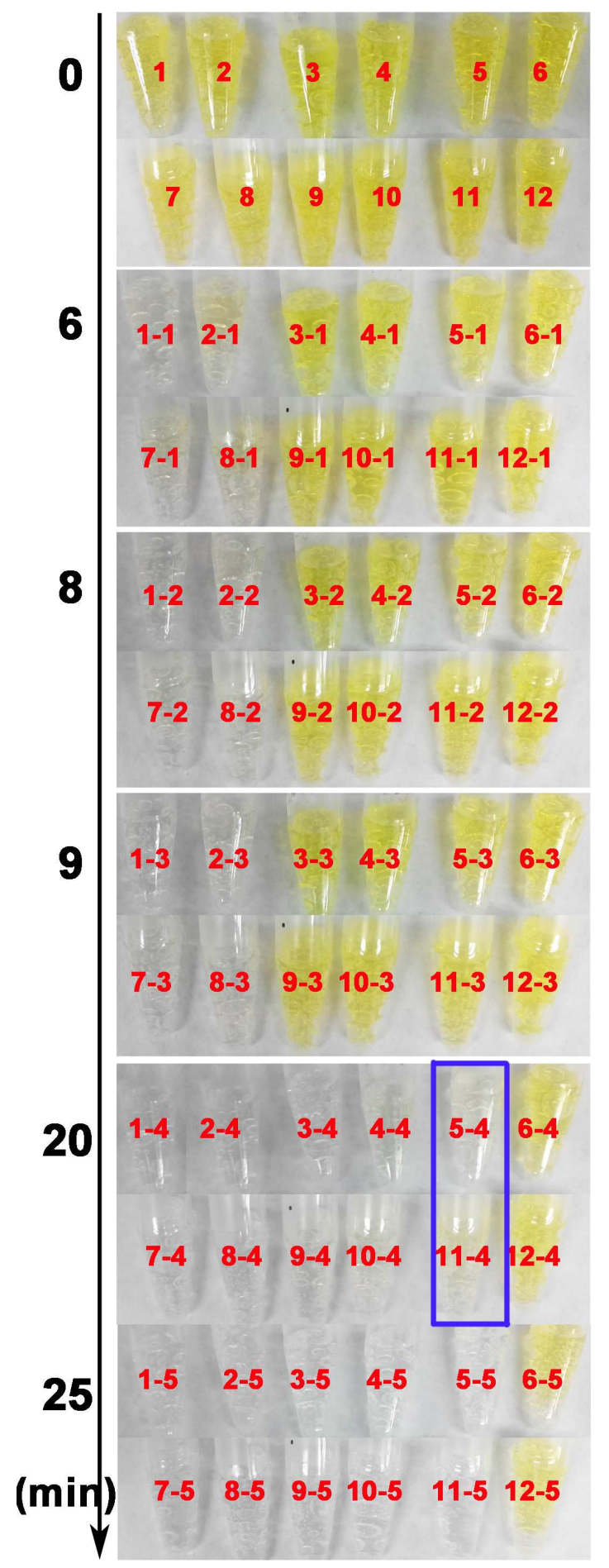

Fig. S7 Optimization of the concentration of AuNPs. 1-6: 2.65nM, 2.05nM, 1.75nM, $1.45 \mathrm{nM}, 1.15 \mathrm{nM}, 0.85 \mathrm{nM}$, the solution without thrombin. $7-12: 2.65 \mathrm{nM}, 2.05 \mathrm{nM}$, $1.75 \mathrm{nM}, 1.45 \mathrm{nM}, 1.15 \mathrm{nM}, 0.85 \mathrm{nM}$, the solution containing thrombin $(0.1 \mathrm{nM})$. 

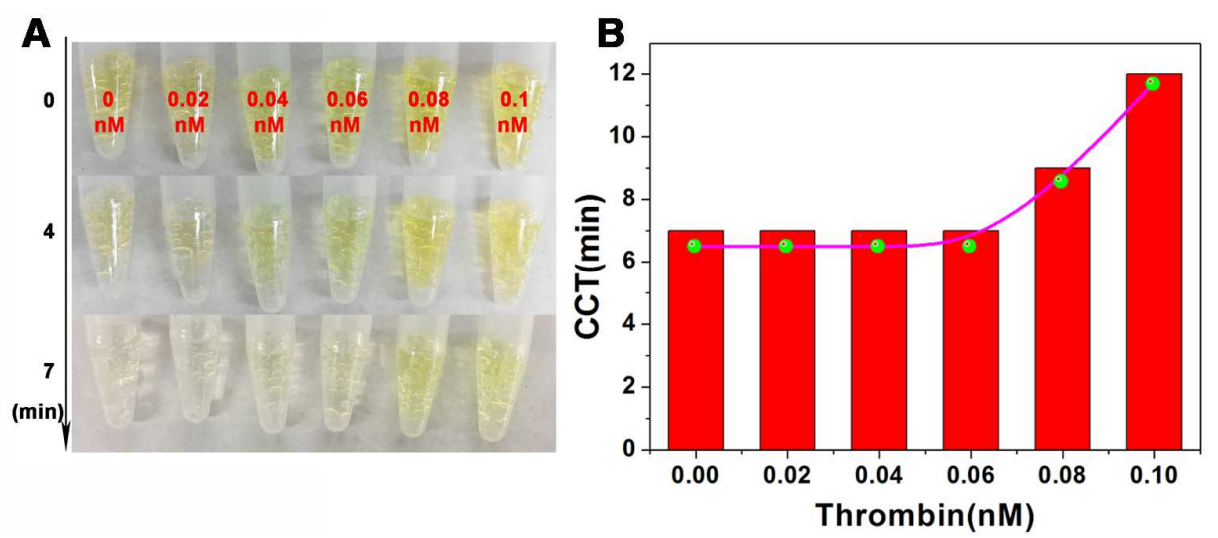

Fig. S8. (A) The visual color changes of the detection mixtures with various concentrations of thrombin $(0,0.02,0.04,0.06,0.08,0.1 \mathrm{nM})$. (B) CCT as a function of varying concentrations of thrombin ranging from 0 to $0.1 \mathrm{nM}$. From Fig.S8, a detection limit of $0.08 \mathrm{nM}$ was obtained. 

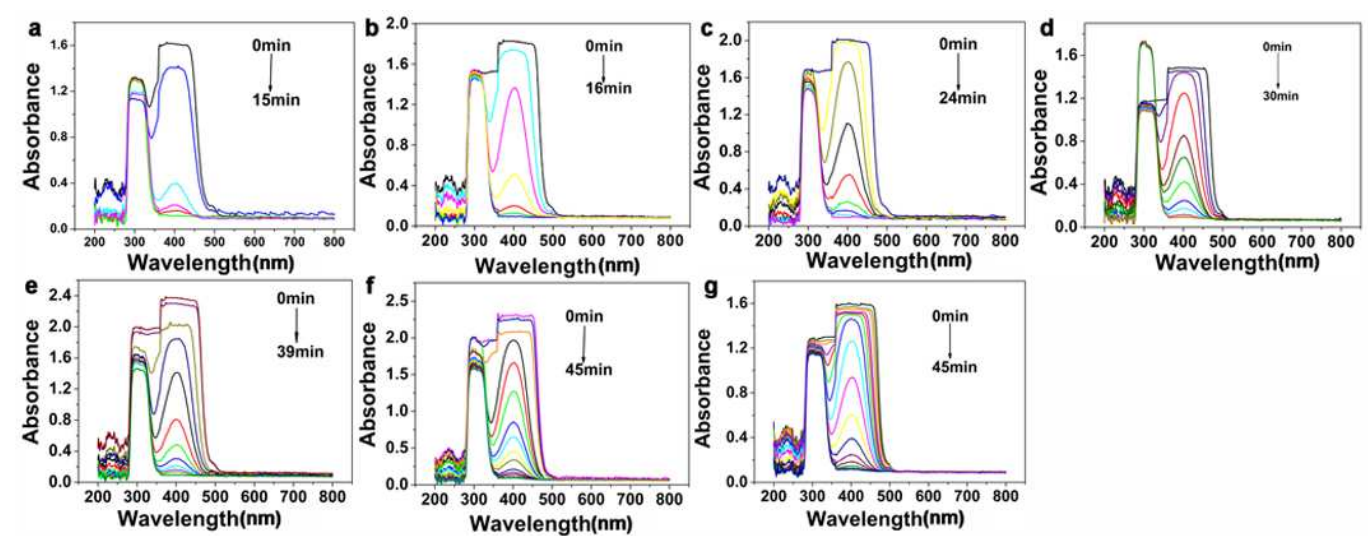

Fig. S9 UV-vis absorption spectra changes of the solution containing thrombin with different concentrations: (a) 0, (b) 0.1, (c) 0.5, (d) 1, (e) 5, (f) 10, and (g) $15 \mathrm{nM}$ showing the catalytic reduction of 4-nitrophenol to 4-aminophenol. 

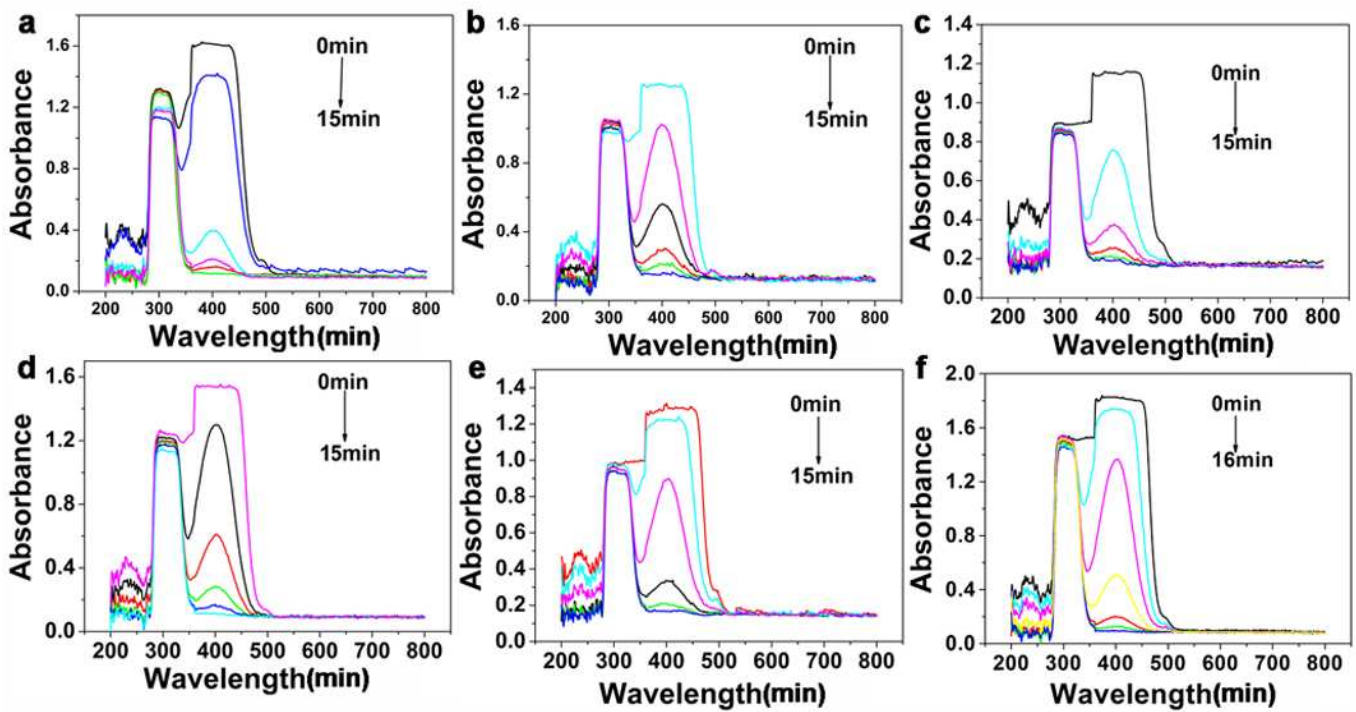

Fig. S10 UV-vis absorption spectra changes of the solution containing thrombin with different concentrations: (a) 0, (b) 0.02, (c) 0.04, (d) 0.06, (e) 0.08, and (f) $0.1 \mathrm{nM}$, showing the catalytic reduction of 4-nitrophenol to 4-aminophenol. 
Table S1 Basic physical properties of proteins.

\begin{tabular}{llc}
\hline protein & MW/kD & pI \\
\hline thrombin (Thr) & 37 & 5.5 \\
lysozeme (Lys) & 14.4 & 11.0 \\
papain (Pap) & 23.0 & 9.6 \\
myoglobin (Myo) & 17.0 & 7.2 \\
hemoglobin (Hem) & 64.5 & 6.8 \\
transferrin (TRF) & 75 & 5.6 \\
human serum albumin (HSA) & 69.4 & 5.2 \\
horseradish peroxidase (HRP) & 40 & 5 \\
bovine serum albumin (BSA) & 66.3 & 4.8 \\
pepsin (Pep) & 35 & $1 \sim 2.5$ \\
\hline
\end{tabular}

